\title{
Editorial
}

\section{Cerebral perfusion and hypothermia}

Extensive clinical experience using profound hypothermic circulatory arrest (PHCA) has suggested that the duration of a "safe" circulatory arrest period is approximately 30-45 minutes. ${ }^{1}$ Recent clinical studies however, have demonstrated a higher incidence of acute, transient postoperative seizures and abnormalities in psychomotor developmental testing one year after a period of PHCA., ${ }^{2,3}$ The implication of these studies are three-fold. One, PHCA imparts a greater neurologic risk to neonates and infants compared with continuous flow bypass. Two, conventional methods of implementing and managing circulatory arrest does not adequately address the quality of perfusion techniques used to prepare the brain prior to PHCA initiation and three, current bypass management strategies do not address cerebral oxygen delivery requirements immediately after a period of PHCA and into the postoperative period.

Protective effects of profound hypothermia on the brain Hypothermia seems to protect the brain from ischemic injury through preserving high energy phosphate stores, preventing excitatory neurotransmitter release, restricting membrane permeability, and preventing calcium entry into the cell. By retarding ischaemic injury, reperfusion can proceed uneventfully.

Several authors have examined the protective effect of deep hypothermia by measuring high energy phosphate compounds with ${ }^{31}$ phosphate nuclear magnetic resonance ( ${ }^{31}$ P-NMR). ${ }^{4-8}$ These authors reported that while ATP was rapidly depleted at normothermic temperatures, ATP levels were maintained at deep hypothermic temperatures $\left(15-20^{\circ} \mathrm{C}\right)$ for a more prolonged period of time. At hypothermic temperatures, the rate of energy dependent cellular enzyme systems such as, Na-K ATPase and CaATPase, are drastically slowed. ATP and phosphocreatine utilization are reduced and ion homeostasis is maintained. However, the level of ATP at which ionic homeostasis is lost is not well defined. Norwood et al., have demonstrated that $25 \mathrm{~min}$ of deep hypothermic arrest sig-

From the Division of Pediatric Cardiac Anesthesia and Critical Care Medicine, Duke University Medical Center, Box 3046 Durham, NC, 27710 USA nificantly lowers creatine phosphate levels, but ATP stores were well maintained, in isolated perfused rat brains. ${ }^{4}$ Sutton et al. and Jonas et al. in separate reports, demonstrate that ATP levels reach their nadir after 21-33 min of hypothermic circulatory arrest. ${ }^{7,8}$ The lose of cellular stores of ATP does not predict the point of cellular injury. Instead it signifies the point at which the energy dependent processes which maintain transmembrane ionic gradients are lost. Deeper levels of hypothermia may provide additional protection by maintaining ion gradients and preventing $\mathrm{Ca}$ entry through altering membrane fluidity, an energy independent process. ${ }^{9}$ At deep hypothermic temperatures, cellular membranes may alter their permeability through changes in physical state of membrane lipids i.e., less liquid and more semisolid. ${ }^{10}$ This may directly retard free ion movement across cellular membranes and prevent acute injury from PHCA. After rewarming from CPB however, the brain is at normothermic temperatures and has normal to increased metabolic demands this may identify a second period of risk.

\section{Global and regional effects of PHCA on cerebral blood flow and cerebral $\mathrm{O}_{2}$ utilization}

In addition to ischemic cellular injury, both clinical and laboratory investigations have documented abnormalities in global measures of cerebral oxygen delivery, cerebral oxygen utilization and cerebral blood flow (CBF) after PHCA. ${ }^{11-14}$ Alterations in cerebral oxygen delivery and cerebral oxygen utilization occur immediately after PHCA, and as suggested by O'Hare and colleagues in this issue of the Canadian Journal of Anaesthesia may persist into the postoperative period. Sustained reductions in CBF or CBFV are most likely secondary to alterations in cerebral oxygen utilization. $12,13,15$

It is well recognized that except during hypothermia, cerebral blood flow remains coupled to cerebral metabolism. During hypothermia, cerebral blood flow decreases linearly with reductions in temperature, while cerebral metabolism decreases exponentially with reductions in temperature. Therefore flow/metabolism ratios must increase with decreasing temperature during CPB in children. In the awake healthy child, and even immediately after CPB with or without PHCA, cerebral blood flow 
(CBF) and metabolism $\left(\mathrm{CMRO}_{2}\right)$ are regulated by the metabolic needs of regional areas of the brain. This is an important regulatory feature of cerebral homeostasis. ${ }^{16-19}$ In humans, a mean CBF of $45-80 \mathrm{ml} \cdot 100$ $\mathrm{gm}^{-1} \cdot \mathrm{min}^{-1}$ is coupled to a $\mathrm{CMRO}_{2}$ of $3.0-4.0 \mathrm{ml} \cdot 100$ $\mathrm{gm}^{-1} \cdot \mathrm{min}^{-1}$, resulting in a $\mathrm{CBF} / \mathrm{CMRO}_{2}$ ratio of 13-20/1. ${ }^{15,20}$ In neonates, $\mathrm{CMRO}_{2}, \mathrm{CBF}$ and the $\mathrm{CBF} /$ $\mathrm{CMRO}_{2}$ ratio are generally higher than older children and adults. This is believed to be due to increased metabolic demand for neuronal growth, myelinization, etc. 21,22 If cerebral metabolism is decreased, cerebral blood flow will also be decreased even under the pathophysiologic conditions associated with prolonged periods of PHCA.

\section{Implications and suggested strategies}

The study by O'Hare et al. in this issue of the Canadian Journal of Anaesthesia identify significant reductions in cerebral blood flow velocity after PHCA and extending well into the postoperative period. Although clinical data describing the effects of PHCA on cerebral oxygen utilization and flow metabolism coupling in the postoperative period have not been reported, the most likely explanation for reduced cerebral blood flow based on current data is alterations in regional brain metabolism. Cerebral flow/metabolism coupling is maintained at normothermic temperatures and has been well described in the immediate post CPB and post PHCA periods. Experimental data in general, supports a strong correlation linking reductions in cerebral blood flow with reductions in cerebral metabolism after PHCA. 8,13,20 Therefore, the important implication of this study is that alterations in regional cerebral blood flow suggest a reduction in cerebral oxygen utilization, and the reduction of cerebral blood flow and oxygen utilization are most likely maintained for at least 4 hours after circulatory arrest.

Attempts to improve cerebral oxygen utilization after PHCA have been evaluated in experimental models. In the prebypass period, experimental data supports improved cooling techniques and the use of NMDA antagonists reduce metabolic disturbances in the immediate postoperative period ${ }^{8,23-25}$ More controversial approaches has been the clinical use of retrograde cerebral perfusion, which has had mixed results in animal studies. ${ }^{26,27}$

In the post-PHCA period the use of modified ultrafiltration, a technique which removes fluid and circulating mediators such as intraleukins, TNF and activated complement C3a and C5a, significantly improves cerebral oxygen utilization after prolonged periods of circulatory arrest in animal models and in humans. ${ }^{28-32}$ The importance of inflammatory mediators underscores the effects of circulatory arrest on cerebral function, in that attempts to increase oxygen delivery by improving oxygen content does not improve cerebral oxygen utilization after PHCA. This finding suggests an important role for inflammatory mediators and supports the notion that reductions in cerebral blood flow and/or attempts to increase cerebral blood flow in the post-operative period may not alter cerebral oxygen utilization.

The prolonged reduction in cerebral blood flow velocity demonstrated by O'Hare and colleagues is an important finding. It suggests that cerebral blood flow and cerebral oxygen utilization remain depressed after PHCA and that the postoperative period remains a high risk period for secondary neurologic injury. Further reductions in cerebral oxygen delivery from systemic reductions in oxygen delivery as may occur with reduction in cardiac output (decreased myocardial function), reduction in oxygen content (due to bleeding, systemic to pulmonary shunting, or $\mathrm{V} / \mathrm{Q}$ mismatch) may be poorly tolerated after PHCA and promote/increase cerebral injury. More over, a reduction in cerebral oxygen delivery may occur due to ventilatory strategies introduced to treat pulmonary artery hypertension, i.e., hyperventilation. Hyperventilation may further reduce cerebral blood flow through primary vasoconstriction after a period of CPB with PHCA. Hyperventilation strategies must therefore be introduced with caution. Alternate therapies such as metabolic alkalization i.e., sodium bicarbonate infusions, and early introduction of nitric oxide etc. as adjunctive therapy may be preferred to minimize the cerebral effects of hyperventilation in patients undergoing profound hypothermic circulatory arrest.

In summary, it becomes increasingly clear that although PHCA is an important technique for improving operating conditions and achieving a high quality repair, it does contribute to cerebral dysfunction and judicious use of PHCA is warranted. When using PHCA, attempts should be made to optimize brain cooling, and minimize the length of time the patient is exposed to PHCA if possible. Recent improvements in brain protection strategies have mitigated some of the potential deleterious effects of PHCA. Further refinements in the way we use PHCA are expected to improve cerebral outcome.

\section{La perfusion cérébrale et l'hypothermie}

En arrêt circulatoire hypothermique profond (ACHP), l'expérience nous a montré que la circulation peut être 
interrompue sans risque indu pendant 30 à $45 \mathrm{~min} .{ }^{1}$ Toutefois, des études cliniques ont récemment fait état de l'augmentation de l'incidence plus élevée de crises convulsives aiguës transitoires et d'anomalies du développement après un an, consécutives à une période d'ACHP. ${ }^{2,3}$ Ces constatations sont importantes pour trois raisons. Premièrement, sous ACHP, le risque de complications neurologiques est plus élevé chez les nouveau-nés et les nourrissons que sous circulation extracorporelle (CEC) à débit continu. Deuxièmement, les méthodes conventionnelles d'initiation et de gestion de l'arrêt circulatoire ne tiennent pas suffisamment compte de la qualité des techniques de perfusion utilisées pour la préparation de cerveau avant l'ACHP, et troisièmement, les stratégies actuelles de gestion de la CEC ne règlent pas les problèmes de transport de l'oxygène au cerveau immédiatement après la période d'ACHP et à la période postopératoire.

\section{La protection du cerveau par lhypothermie profonde}

Il semble que lhypothermie protège le cerveau contre la lésion ischémique en conservant les réserves de phosphates riches en énergie, en prévenant la libération des neurotransmetteurs de l'excitation, en contrôlant la perméabilité de la membrane et en empêchant l'entrée du calcium dans la cellule. En faisant retarder la lésion ischémique, il est possible de reperfuser sans difficultés.

Plusieurs auteurs ont étudié les effets protecteurs de lhypothermie profonde en mesurant les phosphates composés riches en énergie par la resonance magnétique au ${ }^{31}$ phosphate ( $\left.{ }^{31} \mathrm{P}-\mathrm{NMR}\right) .{ }^{4-8}$ Ces auteurs ont rapporté que les niveaux d'ATP se maintenaient plus longtemps en hypothermie profonde $\left(1-20^{\circ} \mathrm{C}\right)$ alors que la déplétion de l'ATP survenait rapidement en normothermie. En hypothermie, la vitesse des systemes enzymatiques cellulaires tels que Na-K ATPase et Ca-ATPase ralentit dramatiquement. L'utilisation de l'ATP et de la phosphocréatine est réduite et l'homéostase ionique maintenue. Cependant, le niveau d'ATP correspondant au moment de l'abolition de lhoméostase ionique est mal connu. Norwood et al. ont montré, sur des cerveau de rats isolés et perfusés, qu'un arrêt circulatoire en hypothermie profonde de 25 minutes diminuait considérablement le niveau de la phosphocréatine mais que les réserves d'ATP étaient maintenues. ${ }^{4}$ Sutton et al. et Jonas et al., dans des communications indépendantes, ont démontré que les niveaux d'ATP peuvent atteindre leur nadir après 21 à $33 \mathrm{~min}$ d'arrêt circulatoire hypothermique. ${ }^{7,8}$ La perte des réserves cellulaires d'ATP ne prédit pas le degré de la lésion cellulaire. Elle indique plutôt à quel moment les mécanismes énergétiques qui maintiennent les gradients ioniques transmembranaires disparaissent. Des niveaux plux profonds dhypothermie peuvent procurer une protection additionnelle en maintenant les gradients ioniques et en prévenant la rentrée du calcium, en altérant la fluidité de la membrane, processus sans dépendance énergétique. ${ }^{9}$ En hypothermie profonde, les membranes cellulaires peuvent altérer leur perméabilité grâce à des modifications de l'tetat physique de la membrane lipidique par ex., en devenant moins liquides et plus semisolides. ${ }^{10}$ Ceci peut retarder le mouvement des ions libres à travers les membranes cellulaires et prévenir ainsi une lésion aiguë due à l'ACHP. Cependant, après réchauffement par la CEC, le cerveau redevient normothermique et a des besoins métaboliques normaux ou plus grands, ce qui peut signifier une seconde période de risque.

\section{Effets globaux et régionaux de l'ACHP sur le débit sanguin cérébral et l'utilisation de l'O, par le cerveau} En plus de l'sischémie cellulaire, des investigations en clinique et en laboratoire ont montré, après l'ACHP, des anomalies portant sur la mesure du transport de l'oxygène au cerveau, sur l'utilisation de l'oxygène cérébral et sur le débit sanguin oérébral (DSC). ${ }^{11-14}$ Les altérations de l'apport en oxygène au cerveau et de son utilisation surviennent immédiatement après l'ACHP, comme le font remarquer O'Hare et al. dans ce numéro du Journal Canadien d'Anesthésie, et elles peuvent persister à la période postopératoire. Les réductions prolongées du DSC sont vraisemblablement secondaires à des altérations de l'utilisation de l'oxygène par le cerveau. ${ }^{12,13,15}$

Il est bien connu qu'à l'exception de la période hypothermique, le débit sanguin demeure couplé au métabolisme cérébral. Pendant lhypothermie, le débit sanguin cérébral diminue de façon linéaire avec le température, alors que le métabolisme cérébral diminue de façon exponentielle avec la température. Par conséquent, le rapport débit/métabolisme augmente chez les enfants avec la baisse de température pendant la CEC. Chez l'enfant éveillé et en bonne santế, et immédiatement après la CEC avec ou sans ACHP, le débit sanguin et le métabolisme cérébral $\left(\mathrm{CMRO}_{2}\right)$ sont contrôlés par les besoins métaboliques des différentes régions du cerveau; ceci est une caractéristique importante de l'homéostase cérébrale. ${ }^{16-19}$ Chez l'humain, en moyenne, un DSC de 45 à $80 \mathrm{ml} \cdot 100$ $\mathrm{mg}^{-1} \cdot \mathrm{min}^{-1}$ est couple avec un $\mathrm{CMRO}_{2}$ de $3,0-4,0$ $\mathrm{ml} \cdot 100 \mathrm{~g}^{-1} \cdot \mathrm{min}^{-1}$, ce qui équivaut à un rapport DSC/ $\mathrm{CMRO}_{2}$ de $13-20 / 1 .{ }^{15,20}$ Chez le nouveau-né, le $\mathrm{CMRO}_{2}$, le $\mathrm{DSC}$ et le rapport $\mathrm{DSC} / \mathrm{CMRO}_{2}$ sont généralement plus élevés que chez l'enfant et l'adultes. On croit que ceci est dû à une augmentation de demande métabolique pour satisfaire aux besoins de la croissance neurale, de la myélinisation, etc. ${ }^{21,22} \mathrm{Si}$ le métabolisme cérébral diminue, le DSC diminuera simultanément, même sous des conditions physiopathologiques propres à des périodes d'ACHP prolongées. 


\section{Conséquences et suggestions stratégiques}

Dans leur travail publié plus loin, O'Hare et de ses collègues ont observé après l'ACHP des réductions importantes de la vélocité du débit sanguin cérébral qui se prolongent pendant la période postopératoire. Bien qülls n'aient pas publié de données cliniques en rapport avec les effets de l'ACHP sur l'utilisation de l'oxygène par le cerveau et sur le couplage du débit/métabolisme à la période postopératoire, selon les données actuelles, la réduction du débit sanguin cérébral est vraisemblablement causée par des altérations du métabolisme cérébral régional. Le couplage débit/métabolisme se maintient en normothermie et a été amplement décrit à la période postCEC immédiate et après des périodes d'ACHP. Les données expérimentales, en général, supportent la solide corrélation qui relie les réductions du débit sanguin cérébral aux réductions du métabolisme cérébral après l'ACHP. ${ }^{8,13,20}$ Par conséquent, les altérations du débit sanguin cérébral régional suggérant une réduction de l'utilisation de l'oxygène par le cerveau constituent l'importante conclusion de cette étude; la réduction du débit sanguin cérébral et de l'utilisation de l'oxygène par le cerveau est vraisemblablement maintenue pour au moins quatre heures après l'arrêt circulatoire.

Des tentatives d'amélioration de l'utilisation de l'oxygène ớrébral après l'ACHP ont été évaluées sur des modèles expérimentaux. A la période pré-CEC, les données expérimentales supportent l'hypothèse selon laquelle l'amélioration des techniques de refroidissement et lutilisation des antagonistes de la NMDA pourraient diminuer les dérangements métaboliques de la période postopératoire immédiate. ${ }^{8,23-25}$ Des approches plus controversées réalisées sur des préparations animales, comme la perfusion cérébrale rétrograde, ont procuré des résultats mitigés. ${ }^{26,27}$

A la période post-ACHP, l'utilisation de l'ultrafiltration modifiée, technique qui retire les liquides et les médiateurs en circulation comme les interleukines, le TNF et les compléments activés C3a et C5a, améliore fortement l'utilisation cérébrale de l'oxygène après des périodes prolongés d'arrêt circulatoire sur des modèles animaux et chez l'humain. ${ }^{28-32}$ L'abondance des médiateurs de l'inflammation accentue les effets de l'arrêt circulatoire sur la fonction cérébrale: l'amélioration du transport de l'oxygène par l'augmentation du contenu n'améliore pas l'utilisation de l'oxygène par le cerveau après l'ACHP. Cette observation accorde un rôle important aux médiateurs de linflammation et supporte la notion que les réductions du débit sanguin cérébral et/ou les tentatives d'augmentation du débit sanguin cérébral à la période postopératoire ne modifieraient en rien l'utilisation de l'oxygène par le cerveau.

La réduction prolongée de la vélocité du débit sanguin cérébral, comme le démontrent O'Hare et al., représente une découverte importante. Elle permet de penser que le débit sanguin cérébral et lutilisation de l'oxygène par le cerveau continuent d'être déprimées après l'ACHP et que la période postopératoire demeure une période à haut risque de lésions neurologiques secondaires. Des réductions supplémentaires de l'apport en oxygène secondaires à des baisses systémiques du transport de l'oxygène comme on le voit au cours d'une chute du débit cardiaque (diminution de l'efficacité myocardique), d'une diminution du contenu en oxygene (par hemorragie, shunt systemique-pulmonaire, ou dysharmonisation du $\mathrm{V} / \mathrm{Q}$ ) peuvent être mal tolérées après un ACHP et favoriser ainsi la lésion cérébrale. En outre, une réduction de l'apport en oxygène peut survenir par des manoeuvres instaures pour traiter l'hypertension pulmonaire, par ex., lhyperventilation. L'hyperventilation peut diminuer davantage le débit sanguin cérébral par vasoconstriction primaire après une période de CEC avec ACHP. L'hyperventilation doit donc être utilisée prudemment. Des manoeuvres de rechange, comme l'alcalinisation métabolique, c.-à-d., les perfusions de bicarbonate de sodium, et l'admission d'oxyde nitrique comme adjuvant, semblent préférables pour minimiser le retentissement cérébral de l'hyperventilation chez les patients qui ont eu un arrêt cardiaque en hyperthermie profonde.

Pour résumer, il devient de plus en plus certain que bien que l'ACHP soit une technique importante pour améliorer les conditions chirurgicales et permettre des réparations de meilleure qualité, il contribue au dysfonctionnement cérébral; il est donc nécessaire de l'utiliser avec discernement. Avec l'ACHP, il faut optimiser le refroidissement cérébral et restreindre au minimum la durée de l'ACHP, lorsque cela est possible. Récemment, des méthodes améliorées de protection cérébrale ont atténué quelques uns des effets nocifs de l'ACHP. Le perfectionnement prévisible de notre façon d'utiliser l'ACHP permettra éventuellement, en ce qui concerne le cerveau, d'améliorer le pronostic.

\section{References}

1 Kirklin JW, Barratt-Boyes BG. Cardiac Surgery. 2nd ed. New York, Edinburgh, London, Melbourne: Churchill Livingstone Inc., 1993; 66-7.

2 Bellinger $D C$, Jonas RA, Rappaport $L A$, et al. Developmental and neurologic sataus of children after heart surgery with hypothermic circulatory arrest or lowflow cardiopulmonary bypass. N Engl J Med 1995; 332: 549-55.

3 Newburger JW, Jonas RA, Wernovsky G, et al. A comparison of the perioperative neurologic effects of hypothermic circulatory arrest versus low-flow cardiopulmonary bypass in infant heart surgery. $\mathrm{N}$ Engl J Med 1993; 329: 1057-64.

4 Norwood WI, Norwood CR, Ingwall JS, Castaneda AR, Fossel ET. Hypothermic circulatory arrest: 31-phosphorus 
nuclear magnetic resonance of isolated perfused neonatal rat brain. J Thorac Cardiovasc Surg 1979; 78: 823-30.

5 Chopp M, Knight $R$, Tidwell CD, Helpern JA, Brown E, Welch $K M$. The metabolic effects of mild hypothermia on global cerebral ischemia and recirculation in the cat: comparison to normothermia and hypothermia. J Cereb Blood Flow Metab 1989; 9: 141-8.

6 Stocker F, Hershkowitz N, Bossi E, et al. Cerebral metabolic studies in situ by 31P-nuclear magnetic resonance after hypothermic circulatory arrest. Ped Res 1986; 20: 867-71.

7 Sutton LN, Clark BJ, Norwood CR, Woodford EJ, Welsh $F A$. Global cerebral ischemia in piglets under conditions of mild and deep hypothermia. Stroke 1991; 22: 1567-73.

8 Jonas $R A$. Experimental studies of hypothermic circulatory arrest and low flow bypass. Cardiol Young 1993; 3: 299-307.

9 Mezrow CK, Gandsas A, Sadeghi AM, et al. Metabolic correlates of neurologic and behavioral injury after prolonged hypothermic circulatory arrest. J Thorac Cardiovasc Surg 1995; 109: 959-75.

10 Rich $T L$, Langer.GA. Calcium depletion in rabbit myocardium. Calcium paradox protection by hypothermia and cation substitution. Circ Res 1982; 51: 131-41.

11 Watanabe T, Orita $H$, Kobayashi $M$, Washio M. Brain tissue $\mathbf{p H}$, oxygen tension, and carbon dioxide tension in profoundly hypothermic cardiopulmonary bypass. J Thorac Cardiovasc Surg 1989; 97: 396-401.

12 Swain JA, McDonald TJ Jr, Griffith PK, Balaban RS, Clark RE, Ceckler $T$. Low flow hypothermic cardiopulmonary bypass protects the brain. J Thorac Cardiovasc Surg 191; 102: 76-84.

13 Greeley WJ, Ungerleider RM, Kern FH, Brusino FG, Smith LR, Reves JG. Effects of cardiopulmonary bypass on cerebral blood flow in neonates, infants and children. Circulation 1989; 80: I 209-15.

14 Greeley WJ, Bushman GA, Kong DL, Oldham HN, Peterson $M B$. Effects of cardiopulmonary bypass on ecosanoid metabolism during pediatric cardiovascular surgery. J Thorac Cardiovasc Surg 1988; 95: 842-9.

15 Murkin JM, Farrar JK, Tweed WA, McKenzie FN, Guiraudon $G$. Cerebral autoregulation and flow/metabolism coupling during cardiopulmonary bypass: the influence of $\mathrm{PaCO}^{2}$. Anesth Analg 1987; 66: 825-32.

16 Stullken EH Jr, Milde JH, Michenfenlder JD, Tinker JH. The non-linear responses of cerebral metabolism to low concentrations of halothane, enflurane, isoflurane and thiopental. Anesth 1977; 46:28-34.

17 Scheinberg $P$, Stead $E A$. The cerebral blood flow in male subjects as measured by the nitrous technique: normal values for blood flow, oxygen utilization, glucose utilization, and peripheral resistance with observations on the effect of lilting and anxiety. J Clin Invest 1949; 28: 1163-8.

18 Kety SS, Schmidt CF. The determination of cerebral blood flow in man by the use of nitrous oxide in low concentrations. Am J Physio 1945; 143: 53-60.

19 Michenfelder JD, Theye RA. Hypothermia: effect of canine brain and whole-body metabolism. Anesthesiology 1968; 29: 1107.

20 Greeley WJ, Kern FH, Ungerleider RM, et al. The effect of hypothermic cardiopulmonary bypass and total circulatory arrest on cerebral metabolism in neonates, infants and children. J Thorac Cardiovasc Surg 1991; 101: 783-94.

21 Julia PL, Kofsky ER, Buckberg GD, Young HH, Bugyi $H I$. Studies of mycardial protection in the immature heart. I. Enhanced tolerance of immature vs adult myocandium to global ischemia with reference to metabolic differences. J Thorac Cardiovasc Surg 1990; 100: 879-8.

22 Rasenberg $A A$, Jones $M D J r$, Traystman RJ, Simmons $M A$, Molteni RA. Response of cerebral blood flow to changes in PCO2 in fetal, newborn and adult sheep. Am J Physiol 1982; 242: H862-6.

23 Kern FH, Jonas RA, Mayer JE Jr, Hanley FL, Casteneda $A R$, Hickey $P R$. Temperature monitoring during $\mathrm{CPB}$ in infants: does it predict efficient brain cooling? Ann Thorac Surg 1992; 54: 749-54.

24 Kern FH, Ungerleider RM, Schulman SR, et al. Comparison of two strategies of CPB cooling on jugular venous oxygen saturation. Anesthesiology 1992; 77: Al136.

25 Mault JR, Ohtake S, Klingensmith ME, Heinle JS, Greeley WJ, Ungerleider RM. Cerebral metabolism and circulatory arrest: effects of duration and strategies for protection. Ann Thorac Surg 1993; 55: 57-63.

26 Lytle BW, McCarthy PM, Meaney KM, Stewart RW, Cosgrove $D M$ 3rd. Systemic hypothermia and circulatory arrest combined with arterial perfusion of the superior vena cava. Effective intraoperative cerebral protection. $J$ Thorac Cardiovasc Surg 1995; 109: 738-43.

27 Yoshimura N, Okada M, Ota T, Nohara H. Pharmacologic intervention for ischemic brain edema after retrograde cerebral perfusion. J Thorac Cardiovasc Surg 1995; 109: 1173-81.

28 Naik SK, Knight A, Elliott MJ A prospective randomized study of a modified technique of ultrafiltration during pediatric open-heart surgery. Circulation 1991; 84(5 suppl): III 422-31.

29 Naik SK, Knight A, Elliott $M J$. A successful modification of ultrafiltration for cardiopulmonary bypass in children. Perfusion 1991; 6: 41-50.

30 Journois D, Pouard P, Greeley WJ, Mauriat P, Vouhé $P$, Safran $D$. Hemofiltration during cardiopulmonary bypass in pediatric cardiac surgery. Effects on hemostasis, cytokines, and complement components. Anesthesiology 1994; 81: 1181-9.

31 Skaryak LA, Kirshbom PM, DiBernardo $L R$ et al. Modified ultrafiltration improves cerebral metabolic recovery after circulatory arrest. J Thorac Candiovasc Surg 1995; 109: 744-51. 